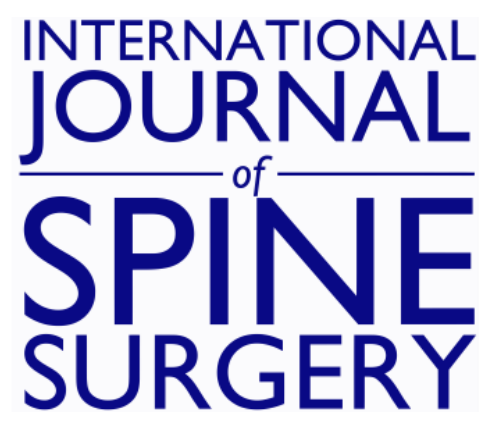

\title{
Long-term Clinical Outcomes Following Endoscopic Foraminoplasty for Patients With Single-Level Foraminal Stenosis of the Lumbar Spine
}

Karlo Houra, Robert Saftic, Emil Klaric and Martin Knight

Int J Spine Surg published online 17 February 2022

http://ijssurgery.com/content/early/2022/02/17/8182

This information is current as of April 26, 2023.

Email Alerts Receive free email-alerts when new articles cite this article. Sign up at:

http://ijssurgery.com/alerts

The International Journal of Spine Surgery

2397 Waterbury Circle, Suite 1,

Aurora, IL 60504, Phone: +1-630-375-1432 


\title{
Long-term Clinical Outcomes Following Endoscopic Foraminoplasty for Patients With Single-Level Foraminal Stenosis of the Lumbar Spine
}

\author{
KARLO HOURA, MD, PHD ${ }^{1,2,3}$; ROBERT SAFTIC, MD, MS ${ }^{1}$; EMIL KLARIC ${ }^{3}$; AND MARTIN KNIGHT, MD, FRCS, \\ $\mathrm{MBBS}^{4}$ \\ ${ }^{I}$ Aksis Specialty Hospital for Spinal and Orthopedic Surgery, Zagreb, Croatia $;{ }^{2}$ University North - University Center Varaždin, Varaždin, Croatia; ${ }^{3}$ School of Medicine, \\ University of Rijeka, Rijeka, Croatia; ${ }^{4}$ The Spinal Foundation, London, UK
}

\begin{abstract}
Background: The aim of this study is to evaluate the safety and long-term clinical outcomes of transforaminal endoscopic foraminoplasty using local anesthesia and total intravenous analgesia (TIVA) in patients with single-level lumbar foraminal stenosis and unilateral leg pain.

Methods: Postoperative pain relief was self-evaluated by 46 consecutive patients using a visual analog scale (VAS) and Oswestry Disability Index (ODI). Patient scores were obtained before the procedure and at 6, 12, 24, and 60 months after surgery.

Results: Pain reduction of at least $50 \%$ in the VAS score and a decrease of at least $50 \%$ or more in ODI score was achieved in 37 of 46 patients throughout the follow-up period. Median VAS score decreased from 7.5 preoperatively to 2.5 postoperatively. Median ODI score decreased from $62 \%$ preoperatively to $15 \%$ postoperatively. All patients reached 24 -month follow-up and 37 patients reached 60-month follow-up. There were no surgery-related complications.

Conclusion: Transforaminal endoscopic foraminoplasty performed under local anesthesia and TIVA produces sustained reduction in pain and improves functionality in patients with single-level lumbar foraminal stenosis without complications even in patients with comorbidities.
\end{abstract}

Clinical Relevance: Endoscopic foraminoplasty may be a useful adjunct to open micro decompressive surgery for patients with foraminal stenosis of the lumbar spine

Level of Evidence: 4.

Endoscopic Minimally Invasive Surgery

Keywords: foraminal stenosis, endoscopic foraminoplasty, lumbar spine

\section{INTRODUCTION}

Segmental foraminal stenosis develops as a result of degenerative changes in the intervertebral disc and facet joints, and it does not just affect elderly patients. It is characterized by the osteoligamentous narrowing of the lateral aspect of the spinal canal and foramen in association with diffuse bulging of the intervertebral disc, vertebral body osteophytosis, hypertrophied articular processes, narrowing of the intervertebral disc height, compression by the superior foraminal ligament, thickened ligamentum flavum or a facet joint capsule, disc herniation, pars defects, facet cysts, and calcification of the foraminal ligaments. ${ }^{1-4}$ Bony foraminal stenosis is aggravated by subluxation of the superior articular process (SAP) in the cranial direction due to SAP subluxation in an anteroposterior direction caused predominantly by intervertebral disc degeneration, facet joint hypertrophy, and facet rotation. These dynamic modalities cause local reactive scarring and tethering of the nerve.

Foraminal irritation or compression causes local Wallerian degeneration of the nerve roots, breakdown of the blood-nerve barrier, increased vascular permeability, and intraradicular edema. ${ }^{5}$ Intraradicular edema leads to increased endoneurial pressure and a form of compartment syndrome. The irritation leads to pain arising from within the surface of the nerve and changes in axon plasma flow. The nerves are themselves innervated by nervi nervorum; during impingement, they may reactively release both substance $\mathrm{P}$ and calcitonin gene-related peptide, thereby amplifying $\mathrm{C}$ nerve fiber nociception. ${ }^{6,7}$ The combination results in back and leg pain and activity-related motor dysfunction.

The conventional surgical treatment is open or miniopen (tubular assisted) paraspinal foraminotomy or total facetectomy combined with fusion surgery. ${ }^{8}$ Percutaneous endoscopic lumbar foraminoplasty was developed 
as an effective minimally invasive foraminal decompression technique for lumbar foraminal stenosis. ${ }^{9,10}$ Transforaminal endoscopic lumbar decompression and foraminoplasty are performed under total intravenous analgesia (TIVA) and local anesthesia, thus allowing older patients with comorbidities and contraindications for general anesthesia to undergo the procedure. The aim of this study is to describe a practical endoscopic foraminoplasty decompression technique for patients suffering from unilateral leg pain and to demonstrate the long-term clinical outcomes from this procedure.

\section{METHODS}

\section{Patients}

Patients' data were prospectively entered into a database, and the records were retrospectively reviewed. Retrospective data were collected from 46 consecutive patients who underwent endoscopic foraminoplasty between January 2008 and January 2018. All patients had failed conservative pain management and physiotherapy for chronic low back and radicular pain. Two experienced neurosurgeons performed the preoperative clinical examinations and surgery. A neurosurgeon, not directly involved in the surgery, conducted the preoperative and postoperative outcome telephone reviews and collated the data for postoperative visual analog scale (VAS) and Oswestry Disability Index (ODI) scores. Approval of the hospital Ethics Committee was obtained, and all patients signed informed consent forms before surgery.

\section{Procedure}

Preoperatively, all patients underwent clinical examination and magnetic resonance imaging (MRI) of the lumbar spine. Foraminal stenosis was confirmed with preoperative sagittal and axial MRI scans. Foraminal stenosis was moderate to severe, with perineural fat obliteration or nerve root compression. ${ }^{11-13}$ All patients had intractable unilateral leg pain for more than 6 months and a self-rated VAS pain score of 5/10 or more . Leg pain was relieved with spinal flexion and was aggravated with spinal extension and physical activity. Patients had neither far lateral or foraminal disc herniations in the spinal canal at the treated level nor axial spinal canal stenosis. All patients underwent failed conservative treatment, such as physical therapy, traction therapy, anti-inflammatory drugs, analgesics, and opioids, as well as 2 selective transforaminal nerve root blocks with different local anesthetic agents. Selective nerve root injections were used as a diagnostic tool to verify the stenotic foramen as the source of leg pain. Patients did not have prior surgery on the target segment. Compressive foraminal stenosis was present only at a single level. Patients who did not benefit from nerve root blocks were not included in this study.

Before the surgery, a neurosurgeon who was not directly involved in the surgery evaluated patients for pain intensity using the VAS and ODI tools. At follow-up reviews at $6,12,24$, and 60 months, the same neurosurgeon conducted the telephone interview for data collection.

\section{Demographic Features}

Over 10 years, we prospectively treated 46 patients (23 men and 23 women; age ranged from 30 to 83 years). All patients had moderate to severe leg pain without back pain. A total of 24 patients had stenosis of the L5 foramen, 15 at the L4 foramen, and 7 patients had stenosis at the L3 foramen.

\section{Surgical Technique}

Patients were operated on in a prone position using local anesthesia and TIVA with biplanar fluoroscopic guidance. ${ }^{14}$ Using preoperative axial magnetic resonance images, a desired trajectory was planned before the surgery for each patient to the target stenotic foramen. A 20G spinal needle with local anesthetic was percutaneously advanced under fluoroscopic guidance toward the SAP or a lower part of the foramen in the safe zone. ${ }^{15}$ A cannulated TOM Shidi bone needle with a diamond tip was inserted through a $1-\mathrm{cm}$ skin incision and was hammered through the anterolateral part of SAP, traversing it and breaching its medial cortex. By performing this maneuvre under fluoroscopic control, the exiting nerve root is protected in the stenotic foramen during the approach.

In the final position, the tip of the TOM Shidi needle was located at the entrance of the disc, but not in the disc, in the lateral view, and at the medial pedicle line in the anteroposterior (AP) view (Figure 1). A Kirchner guide wire was then introduced through the TOM Shidi bone needle, and the transforaminal trajectory for the endoscope to access the stenotic foramen was established. The paraspinal muscles were dilatated using tapered, cannulated dilators passed over the guide wire (Figure 2). Enlargement of the neural foramen was accomplished with serial blunt-tipped manual reamers. All reamers were advanced over the guide wire under $\mathrm{x}$-ray control (Figure 3). This technique facilitates initial entry into the foramen compared to undercutting of the SAP under direct vision after introducing an endoscope. 

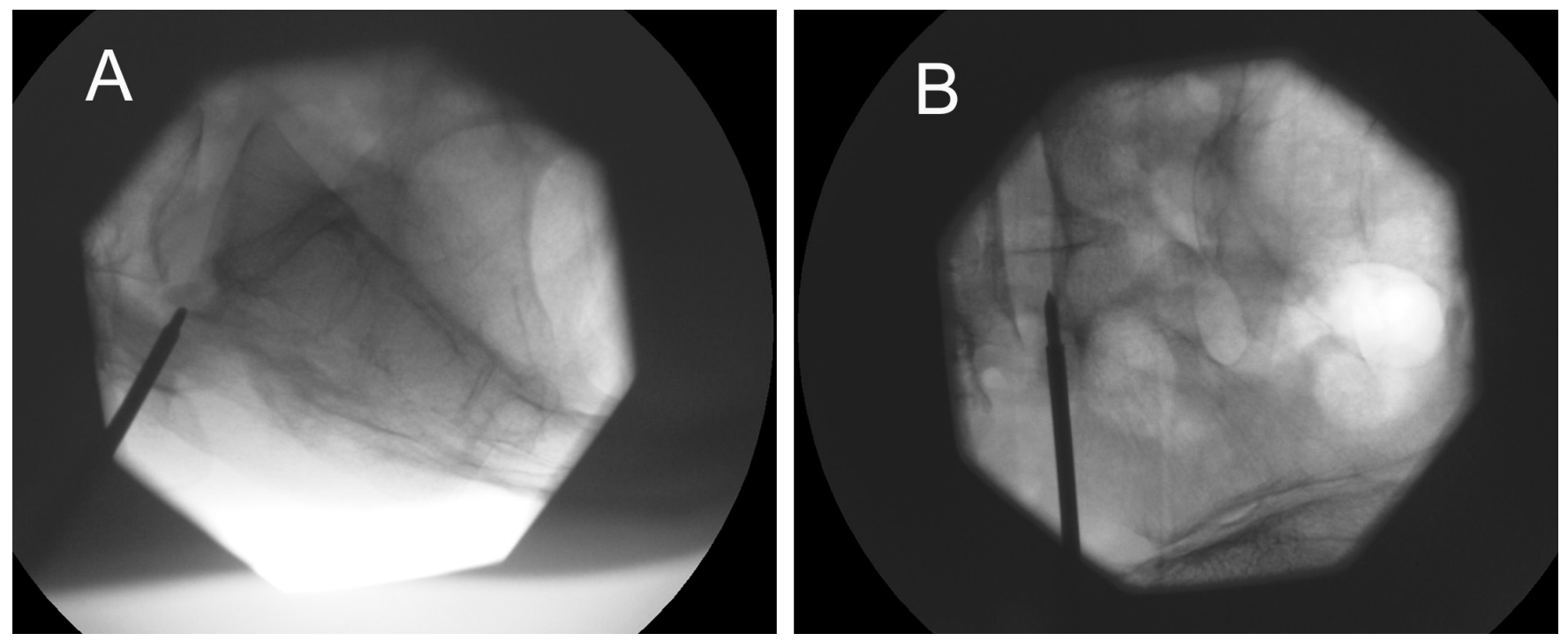

Figure 1. (A) Intraoperative fluoroscopic lateral view of the lower lumbar spine showing TOM Shidi needle advancement through the superior articular process of S1 vertebra in the lower part of the L5 foramen on the left side. (B) Intraoperative fluoroscopic anteroposterior view of the lower lumbar spine showing a final position of TOM Shidi needle with its tip at the medial pedicle line.

By operating using TIVA, the patient's safety is assured because the patient will alert the surgeon promptly with pain or discomfort if the reamer is too close to the nerve. If this happens, the surgical approach is altered and the direct foraminal approach is utilized. This involves inserting a bevel-ended working cannula over the muscle dilatator with the sharp end directed toward the safe working zone, snugging under the SAP and medial to the nerve root (Figure 4). In this case, foraminal undercutting is performed under direct

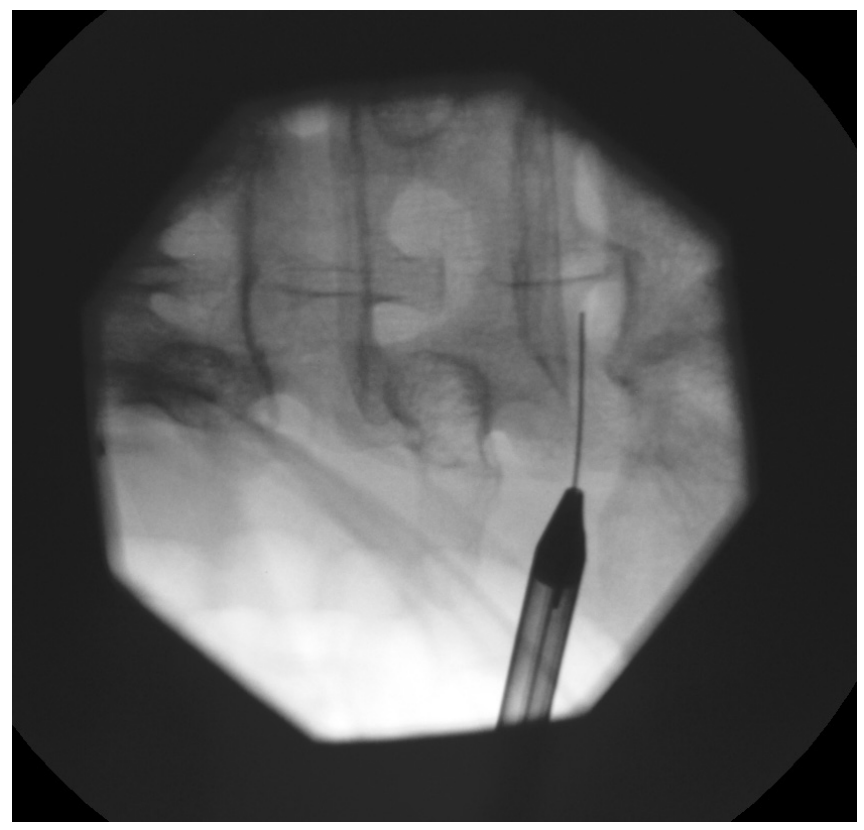

Figure 2. Intraoperative fluoroscopic anteroposterior view of the lower lumbar spine showing dilatation of the paraspinal muscles using cannulated dilator passed over the guide wire. endoscopic vision using straight or curved electric burrs, which lessens the risk of SAP fracture ${ }^{16}$ (Figure 5). The typical direction of bone removal with either technique migrates from caudal to cranial and medially until the apex of the SAP and epidural space is reached. Foraminal undercutting should be performed until the surgeon reaches the ligamentum flavum or a soft tissue. ${ }^{17}$ The bevel end of the working cannula can be used as a useful neural retractor by rotating the cannula. To complete the decompression, the tip and the cranial part of SAP, the thickened ligamentum flavum, superior foraminal ligament, and fibrotic adhesions surrounding the nerve need to be removed using dissecting probes and endoscopic punches until the pulsatility of the exiting and traversing nerve has been restored. The surgeon should always confirm the position of the exiting nerve root during the entire procedure. The nerve needs to be mobilized from the vertebra and disc and shoulder osteophytes removed. A radiofrequency probe is used to control the epidural bleeding. ${ }^{18}$ A curved dissecting probe is used to dissect scar tissues and to additionally confirm the decompression status. The endpoint of the procedure is achieved with the free mobilization of the exiting nerve root, and the pulsations of the exiting nerve root and dural sac are restored. ${ }^{16,19,20}$ The decompression should cover the entire course of the exiting nerve root from the axilla throughout the extraforaminal exit zone (Figure 6) and ensure adequate medial foraminal undercutting. This extremely difficult to achieve at the L5/S1 level. The procedure usually takes between 60 and 80 minutes in experienced surgeons' hands. Patients were discharged home within 24 hours after the surgery. 

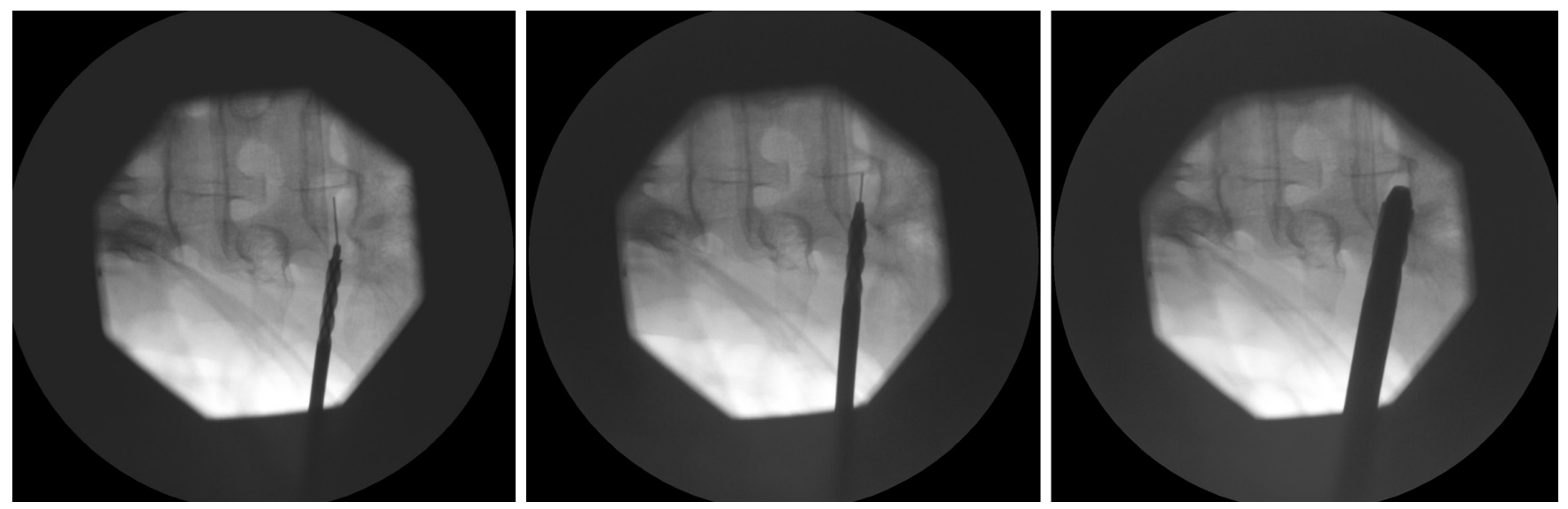

Figure 3. Intraoperative fluoroscopic anteroposterior view of the lower lumbar spine showing various sizes of blunt tip hand reamers used for bony decompression introduced into foramen L5 over the guidewire.

\section{Statistical Analysis}

The VAS for pain and the ODI values were analyzed using mixed modeling. VAS or ODI was defined as dependent variable, while factors, such as level of the foraminal stenosis (L2, L3, L4, and L5), timepoint (pre and post) nested within consistency, and months (levels $0,6,12,24$, and 60) nested within timepoint, were set as fixed factors. Sex and age were also included as fixed factors. Subject identifier was set as random intercept, and age was set as random slope. Prior modeling, VAS, and ODI were transformed by inverse transformation of ranks to standard normal distribution. In total, 42 post hoc tests were applied (21 per index) to estimate the difference between the levels of foraminal stenosis (3); after and before treatment for each level of foraminal stenosis (3); differences (after and before) between the levels of stenosis (3); and consecutive months levels in the posttreatment period ( 6 vs 0,12 vs 6,24 vs 12,60 vs 24) for each level of stenosis (12). False discovery rate was controlled using Benjamini-Hochberg method, and only adjusted $P$ values were reported. All statistical analyses were computed using $\mathrm{R}$ software for statistical computing, version 3.6.3. (R Core Team, Vienna, Austria).

\section{RESULTS \\ Operative Findings}

Sufficient decompression was achieved in all cases. Intraoperatively, restoration of the pulsating dura mater together with free mobilization of the exiting nerve root was taken as direct evidence of sufficient decompression
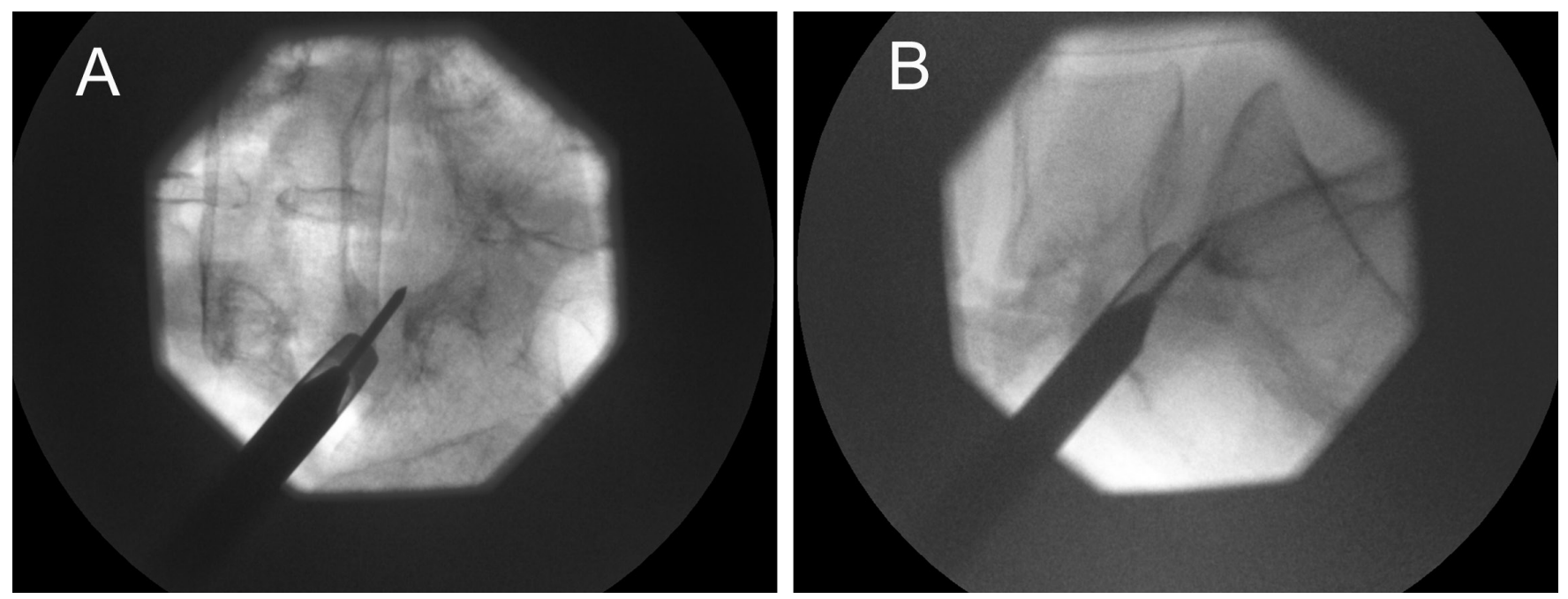

Figure 4. (A) Intraoperative fluoroscopic anteroposterior view of the lower lumbar spine showing bevel-ended working cannula over the muscle dilatator with the sharp end directed toward the safe working zone, snugging under the superior articular process (SAP) and medial to the nerve root. (B) Intraoperative fluoroscopic lateral view of the lower lumbar spine showing bevel-ended working cannula over the muscle dilatator with the sharp end directed toward the safe working zone, snugging under the SAP and medial to the nerve root. 


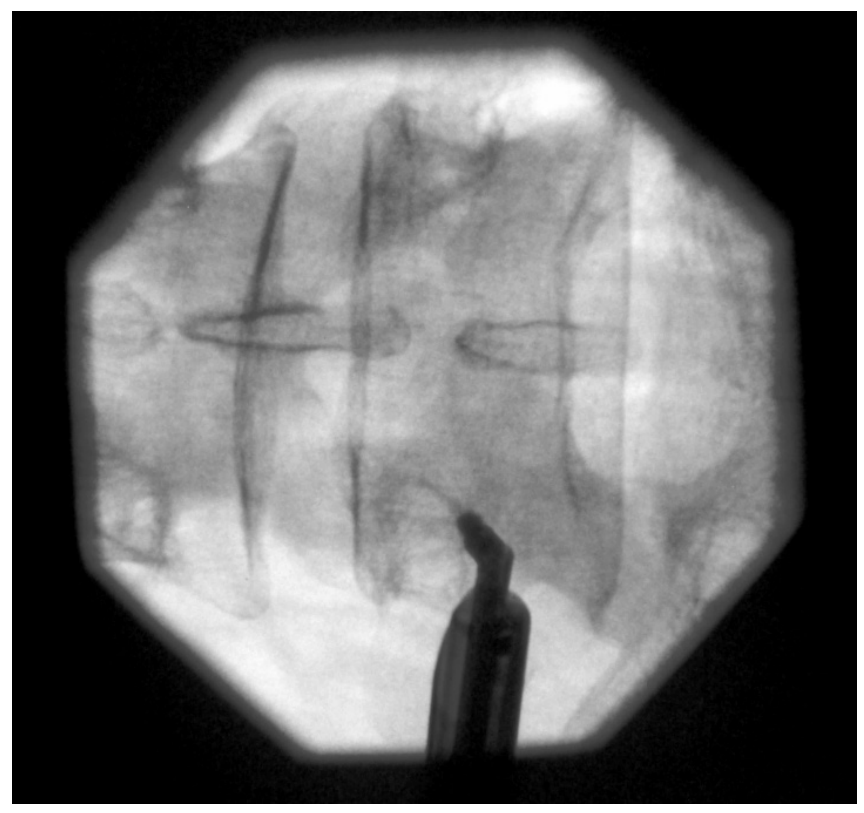

Figure 5. Intraoperative fluoroscopic anteroposterior view of the lower lumbar spine showing the position of the electric burr with curved tip used for bone drilling of teh caudal part of L5 pedicle to adequately decompress the exiting nerve root.

during surgery. Postoperative MRI images of the lumbar spine confirmed that compression and distortion of the nerve and its pathway had been relieved and that the nerve was surrounded by free space along its pathway to the external foraminal portal and therefore decompression had been achieved. All patients tolerated endoscopic surgery well, without serious postsurgical pain or neurological sequelae.

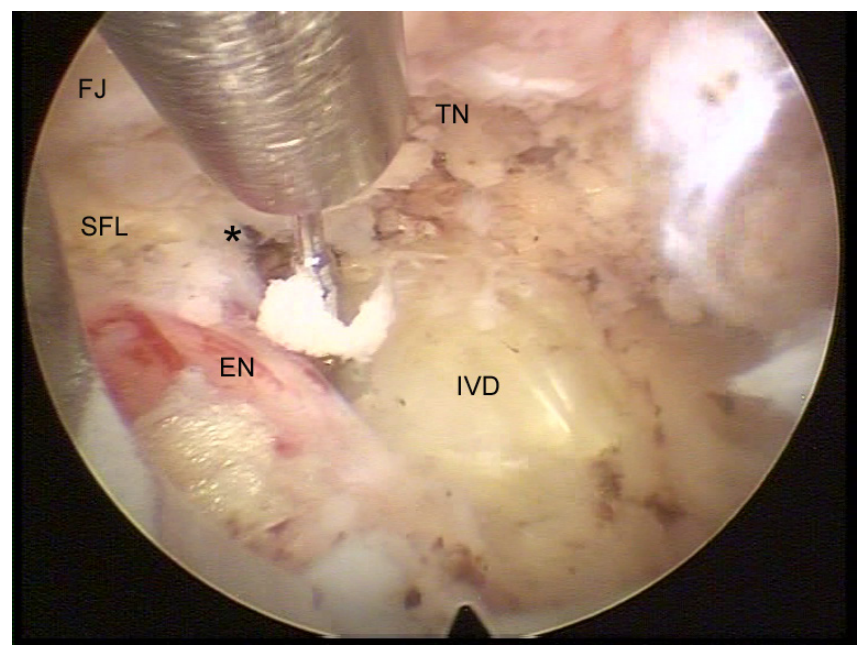

Figure 6. Intraoperative view showing decompression of L5 nerve root in the left foramen. Blunt tip probe is used for liberation of the nerve root from the adhesions to the disc. The superior foraminal ligament (SFL) should be cut to fully accomplish and maintain adequate foraminoplasty and pulsatility of the nerve. EN, exiting nerve root; FJ, facet joint; IVD, intervertebral disc; TN, descending nerve, ${ }^{*}$, axilla.

\section{Patient Outcome Scores}

Forty-six patients were included in this study. The patients' demographics and preoperative and postoperative VAS scores are presented in Table 1. The patients' demographics and preoperative and postoperative ODI scores are presented in Table 2. All patients reached 24month follow-up, and 37 patients reached 60-month follow-up. Reduction of more than $50 \%$ points in the VAS score and more than 50\% in the ODI are considered "relevant," $16,21,22$ which provides a notional surgical success rate of $37 / 46(80.43 \%)$.

The data presented in Figures 7 and 8 show the following:

1. Thirty-seven of 46 patients achieved a postoperative decrease of at least $50 \%$ in the VAS points and a decrease of at least 50\% in their ODI scores postoperatively.

2. ODI scores closely matched the improvement in VAS scores.

3. The benefit, following endoscopic foraminoplasty, that was secured at the 6 months postoperative review was sustained throughout the longer follow-up.

4. Patients who failed to achieve the "relevant" decrease in VAS scores at 6 months from surgery continued to have intermittent pain of moderate severity throughout the follow-up period.

5. There was no statistically significant difference in postoperative functional outcome or pain scores between the levels of foraminal stenosis.

6. Stenosis most commonly occurred at L5 foramen, followed by L4 and L3 foraminae.

\section{DISCUSSION}

\section{Analysis of Good and Poor Outcomes}

Yeung and Gore $^{23}$ treated 30 patients using the same surgical technique under local anesthesia. The mean VAS score in these patients improved from 7.2 preoperatively to 4.0 postoperatively. Our patients show slightly better results, with a mean preoperative VAS score of 7.47 that dropped to 3.41 postoperatively at 24-month follow-up. In their study, ${ }^{23}$ ODI decreased from $48 \%$ to $31 \%$; in our results, preoperative ODI was $58.65 \%$ and dropped to $25.52 \%$ postoperatively at 24-month follow-up. Lewandrowski, ${ }^{24,25}$ who included disc herniations and bone stenosis, reported excellent and good results in $75 \%$ of patients with bony foraminal stenosis using MacNub criteria, ODI, and VAS scores. The success rate in our study was slightly higher at $80.43 \%$. Our results are also comparable to those 
Table 1. The demographics, level of foraminal stenosis, preoperative, and postoperative (in months) visual analog scale scores (0-10) for each consecutive patient.

\begin{tabular}{|c|c|c|c|c|c|c|c|}
\hline Case Number & Sex/Age, $y$ & Level of Foraminal Stenosis & Pre-VAS & $\begin{array}{c}\text { Post-VAS } \\
6 \mathrm{mo} \\
\end{array}$ & $\begin{array}{c}\text { Post-VAS } \\
12 \mathrm{mo} \\
\end{array}$ & $\begin{array}{c}\text { Post-VAS } \\
24 \text { mo } \\
\end{array}$ & $\begin{array}{c}\text { Post } \\
\text { VAS } \\
60 \mathrm{mo} \\
\end{array}$ \\
\hline 1 & $\mathrm{M} / 61$ & L5 & 8 & 3 & 2 & 2 & 2 \\
\hline 2 & $\mathrm{M} / 63$ & L5 & 7 & 3 & 2 & 2 & 2 \\
\hline 3 & $\mathrm{M} / 79$ & L3 & 7 & 3 & 3 & 3 & 3 \\
\hline 4 & $\mathrm{M} / 39$ & L5 & 7 & 3 & 3 & 3 & 3 \\
\hline 5 & $\mathrm{~F} / 30$ & L5 & 9 & 4 & 4 & 4 & 4 \\
\hline 6 & $\mathrm{~F} / 69$ & L4 & 7 & 8 & 8 & 8 & 8 \\
\hline 7 & $\mathrm{~F} / 69$ & L4 & 7 & 2 & 0 & 0 & 0 \\
\hline 8 & $\mathrm{~F} / 48$ & L5 & 8 & 4 & 4 & 4 & 4 \\
\hline 9 & $\mathrm{~F} / 51$ & L5 & 6 & 2 & 2 & 1 & 1 \\
\hline 10 & $\mathrm{~F} / 75$ & L5 & 6 & 2 & 2 & 2 & 2 \\
\hline 11 & $\mathrm{~F} / 47$ & L4 & 8 & 7 & 7 & 7 & 7 \\
\hline 12 & $\mathrm{M} / 64$ & L3 & 7 & 7 & 7 & 7 & 7 \\
\hline 13 & $\mathrm{~F} / 61$ & L5 & 8 & 2 & 1 & 1 & 1 \\
\hline 14 & $\mathrm{M} / 61$ & L4 & 6 & 2 & 2 & 2 & 2 \\
\hline 15 & $\mathrm{~F} / 64$ & L4 & 8 & 4 & 3 & 3 & 3 \\
\hline 16 & $\mathrm{M} / 58$ & L4 & 6 & 5 & 5 & 5 & 5 \\
\hline 17 & $\mathrm{~F} / 68$ & L4 & 7 & 3 & 3 & 3 & 3 \\
\hline 18 & $\mathrm{~F} / 46$ & L5 & 8 & 3 & 2 & 2 & 2 \\
\hline 19 & $\mathrm{M} / 46$ & L5 & 8 & 4 & 4 & 4 & 4 \\
\hline 20 & $\mathrm{M} / 83$ & L4 & 7 & 6 & 6 & 6 & 6 \\
\hline 21 & $\mathrm{~F} / 47$ & L5 & 6 & 2 & 2 & 1 & 1 \\
\hline 22 & $\mathrm{~F} / 37$ & L5 & 8 & 7 & 7 & 7 & 7 \\
\hline 23 & F/64 & L5 & 6 & 3 & 2 & 2 & 2 \\
\hline 24 & $\mathrm{M} / 51$ & L5 & 9 & 3 & 2 & 2 & 2 \\
\hline 25 & $\mathrm{~F} / 55$ & L5 & 9 & 2 & 0 & 0 & 0 \\
\hline 26 & M/69 & L3 & 7 & 3 & 3 & 2 & 2 \\
\hline 27 & $\mathrm{~F} / 52$ & L5 & 9 & 4 & 4 & 4 & 4 \\
\hline 28 & $F / 62$ & L4 & 10 & 3 & 2 & 2 & 2 \\
\hline 29 & $\mathrm{M} / 42$ & L5 & 7 & 3 & 2 & 2 & 2 \\
\hline 30 & $\mathrm{M} / 68$ & L3 & 6 & 2 & 1 & 1 & 1 \\
\hline 31 & $\mathrm{~F} / 46$ & L3 & 9 & 3 & 3 & 3 & 3 \\
\hline 32 & $\mathrm{~F} / 80$ & L5 & 6 & 3 & 2 & 2 & 2 \\
\hline 33 & $\mathrm{M} / 48$ & L3 & 7 & 3 & 3 & 3 & 3 \\
\hline 34 & $\mathrm{M} / 71$ & L5 & 8 & 8 & 8 & 8 & 8 \\
\hline 35 & $\mathrm{M} / 74$ & L4 & 8 & 3 & 2 & 2 & 2 \\
\hline 36 & F/69 & L5 & 7 & 3 & 3 & 3 & 3 \\
\hline 37 & $\mathrm{M} / 41$ & L5 & 6 & 3 & 2 & 2 & 2 \\
\hline 38 & $\mathrm{M} / 75$ & L4 & 8 & 4 & 3 & 3 & \\
\hline 39 & $\mathrm{M} / 71$ & L4 & 8 & 7 & 7 & 7 & \\
\hline 40 & $\mathrm{M} / 42$ & L4 & 7 & 7 & 7 & 7 & \\
\hline 41 & $\mathrm{~F} / 74$ & L4 & 8 & 3 & 2 & 2 & \\
\hline 42 & $\mathrm{M} / 41$ & L4 & 7 & 2 & 2 & 1 & \\
\hline 43 & $\mathrm{~F} / 64$ & L5 & 8 & 4 & 4 & 3 & \\
\hline 44 & $\mathrm{M} / 72$ & L5 & 8 & 3 & 2 & 2 & \\
\hline 45 & $\mathrm{~F} / 83$ & L3 & 8 & 3 & 3 & 2 & \\
\hline 46 & $\mathrm{M} / 75$ & L5 & 9 & 4 & 4 & 4 & \\
\hline
\end{tabular}

Abbreviation: VAS, visual analog scale.

published by Ahn et al, ${ }^{10}$ who reported an improvement in mean VAS score for leg pain, from 8.36 at baseline to 1.97 at 2 years postsurgery, and mean ODI score, from 65.8 at baseline to 19.3 at 2 years postsurgery.

Ahn et $\mathrm{al}^{10,26}$ and Yeung and Gore ${ }^{23}$ followed their patients for 2 years while Lewandrowski had a mean follow-up period of 33 and 46 months in his 2 published articles. ${ }^{24,25}$ In our study, 37 patients completed long-term follow-up of 5 years, but statistical analysis showed that all patients who accomplished good recovery after surgery in the first 2 years maintained good results through the end of 5-year follow-up (Figures 7 and 8). With this in mind, we can expect that for the 7 patients who scored well in the first 2 years postoperatively but did not yet complete a 5-year follow-up, their outcomes will probably remain positive. Therefore, this study demonstrated that if the patient is compliant with postoperative rehabilitation, then the benefits achieved at 6 months are sustained at 5 years postoperatively in 37 of 46 patients $(80.43 \%)$.

\section{"Poor" and "Worse" Results}

One patient with L4 foraminal stenosis failed to achieve significant pain reduction based on VAS and ODI scores following surgery. She reported slightly increased symptoms through all follow-up periods; thus, her outcome was 
Table 2. The demographics, level of foraminal stenosis, preoperative, and postoperative (in months) Oswestry Disability Index scores (0-100) for each consecutive patient.

\begin{tabular}{|c|c|c|c|c|c|c|c|}
\hline Case Number & Sex/Age, y & Level of Foraminal Stenosis & Pre-ODI & $\begin{array}{c}\text { Post-ODI } \\
6 \mathrm{mo}\end{array}$ & $\begin{array}{c}\text { Post-ODI } \\
12 \mathrm{mo}\end{array}$ & $\begin{array}{c}\text { Post-ODI } \\
24 \text { mo }\end{array}$ & $\begin{array}{l}\text { Post-ODI } \\
60 \mathrm{mo}\end{array}$ \\
\hline 1 & $\mathrm{M} / 61$ & L5 & 70 & 12 & 6 & 6 & 6 \\
\hline 2 & $\mathrm{M} / 63$ & L5 & 48 & 10 & 2 & 2 & 2 \\
\hline 3 & $\mathrm{M} / 79$ & L3 & 48 & 12 & 8 & 8 & 8 \\
\hline 4 & $\mathrm{M} / 39$ & L5 & 40 & 16 & 14 & 14 & 14 \\
\hline 5 & $\mathrm{~F} / 30$ & L5 & 86 & 42 & 38 & 38 & 38 \\
\hline 6 & $\mathrm{~F} / 69$ & L4 & 54 & 76 & 76 & 76 & 76 \\
\hline 7 & $\mathrm{~F} / 69$ & L4 & 66 & 6 & 2 & 2 & 2 \\
\hline 8 & $\mathrm{~F} / 48$ & L5 & 56 & 26 & 26 & 26 & 26 \\
\hline 9 & $\mathrm{~F} / 51$ & L5 & 44 & 12 & 8 & 8 & 8 \\
\hline 10 & $\mathrm{~F} / 75$ & L5 & 26 & 6 & 2 & 2 & 2 \\
\hline 11 & $\mathrm{~F} / 47$ & $\mathrm{~L} 4$ & 58 & 52 & 52 & 52 & 52 \\
\hline 12 & $\mathrm{M} / 64$ & L3 & 40 & 40 & 40 & 40 & 40 \\
\hline 13 & $\mathrm{~F} / 61$ & L5 & 58 & 4 & 4 & 4 & 4 \\
\hline 14 & $\mathrm{M} / 61$ & L4 & 48 & 10 & 8 & 8 & 8 \\
\hline 15 & $\mathrm{~F} / 64$ & L4 & 64 & 30 & 28 & 28 & 28 \\
\hline 16 & $\mathrm{M} / 58$ & L4 & 38 & 32 & 32 & 32 & 32 \\
\hline 17 & $\mathrm{~F} / 68$ & L4 & 66 & 20 & 16 & 16 & 16 \\
\hline 18 & $\mathrm{~F} / 46$ & L5 & 64 & 6 & 4 & 4 & 4 \\
\hline 19 & $\mathrm{M} / 46$ & L5 & 64 & 30 & 30 & 30 & 30 \\
\hline 20 & $\mathrm{M} / 83$ & L4 & 70 & 64 & 64 & 64 & 64 \\
\hline 21 & $\mathrm{~F} / 47$ & L5 & 50 & 8 & 6 & 6 & 6 \\
\hline 22 & $\mathrm{~F} / 37$ & L5 & 66 & 60 & 60 & 60 & 60 \\
\hline 23 & $\mathrm{~F} / 64$ & L5 & 46 & 22 & 18 & 18 & 18 \\
\hline 24 & $\mathrm{M} / 51$ & L5 & 74 & 16 & 14 & 14 & 14 \\
\hline 25 & $\mathrm{~F} / 55$ & L5 & 72 & 6 & 2 & 2 & 2 \\
\hline 26 & $\mathrm{M} / 69$ & L3 & 56 & 16 & 12 & 12 & 12 \\
\hline 27 & $\mathrm{~F} / 52$ & L5 & 74 & 36 & 36 & 36 & 36 \\
\hline 28 & $\mathrm{~F} / 62$ & L4 & 80 & 18 & 14 & 14 & 14 \\
\hline 29 & $\mathrm{M} / 42$ & L5 & 56 & 14 & 10 & 10 & 10 \\
\hline 30 & $\mathrm{M} / 68$ & L3 & 40 & 8 & 4 & 4 & 4 \\
\hline 31 & $\mathrm{~F} / 46$ & L3 & 78 & 26 & 24 & 24 & 24 \\
\hline 32 & $\mathrm{~F} / 80$ & L5 & 42 & 18 & 16 & 16 & 16 \\
\hline 33 & $\mathrm{M} / 48$ & L3 & 70 & 34 & 34 & 34 & 34 \\
\hline 34 & $\mathrm{M} / 71$ & L5 & 62 & 60 & 60 & 60 & 60 \\
\hline 35 & $\mathrm{M} / 74$ & L4 & 66 & 18 & 14 & 14 & 14 \\
\hline 36 & $\mathrm{~F} / 69$ & L5 & 56 & 28 & 28 & 28 & 28 \\
\hline 37 & $\mathrm{M} / 41$ & L5 & 48 & 14 & 10 & 10 & 10 \\
\hline 38 & $\mathrm{M} / 75$ & L4 & 62 & 30 & 28 & 28 & \\
\hline 39 & $\mathrm{M} / 71$ & L4 & 64 & 60 & 60 & 60 & \\
\hline 40 & $\mathrm{M} / 42$ & L4 & 50 & 48 & 48 & 48 & \\
\hline 41 & $\mathrm{~F} / 74$ & $\mathrm{~L} 4$ & 64 & 10 & 6 & 6 & \\
\hline 42 & $\mathrm{M} / 41$ & L4 & 52 & 12 & 8 & 8 & \\
\hline 43 & $\mathrm{~F} / 64$ & L5 & 64 & 28 & 26 & 26 & \\
\hline 44 & $\mathrm{M} / 72$ & L5 & 66 & 18 & 14 & 14 & \\
\hline 45 & $\mathrm{~F} / 83$ & L3 & 62 & 20 & 16 & 16 & \\
\hline 46 & $\mathrm{M} / 75$ & L5 & 70 & 32 & 32 & 32 & \\
\hline
\end{tabular}

Abbreviation: ODI, Oswestry Disability Index.

defined as "worse." The exact reason for the pain exacerbation is unclear; however, there are several possible explanations. This patient was diabetic and obese (BMI 30). She presented with a history of foraminal stenosis for more than 10 years. Also, this patient underwent surgery in the early period of the authors' learning curve, so aggressive manipulation of dorsal root ganglion of the exiting spinal nerve may explain the unfavorable outcome. The pattern of her outcome was one of gradual deterioration and may reflect the development of perineural scarring or a disc degeneration. One year after endoscopic foraminoplasty, she underwent fusion surgery in another hospital but thereafter still complained of severe leg pain.
Eight patients failed to achieve a "relevant" benefit and were deemed to have a "poor" outcome. Although they reported a satisfactory decrease in leg pain, they had developed low back pain postoperatively. Their outcome may be explained by the development of the annular fissures, degenerative changes of the intervertebral disc, facet joints, or compression to the posterior longitudinal ligament caused by disc bulging or herniation. For these patients, endoscopic radiofrequency neuroablation of pain generators in the annulus, facet joints or cluneal nerve trigger point irritation, or a fusion surgery might be an option. ${ }^{27}$ 

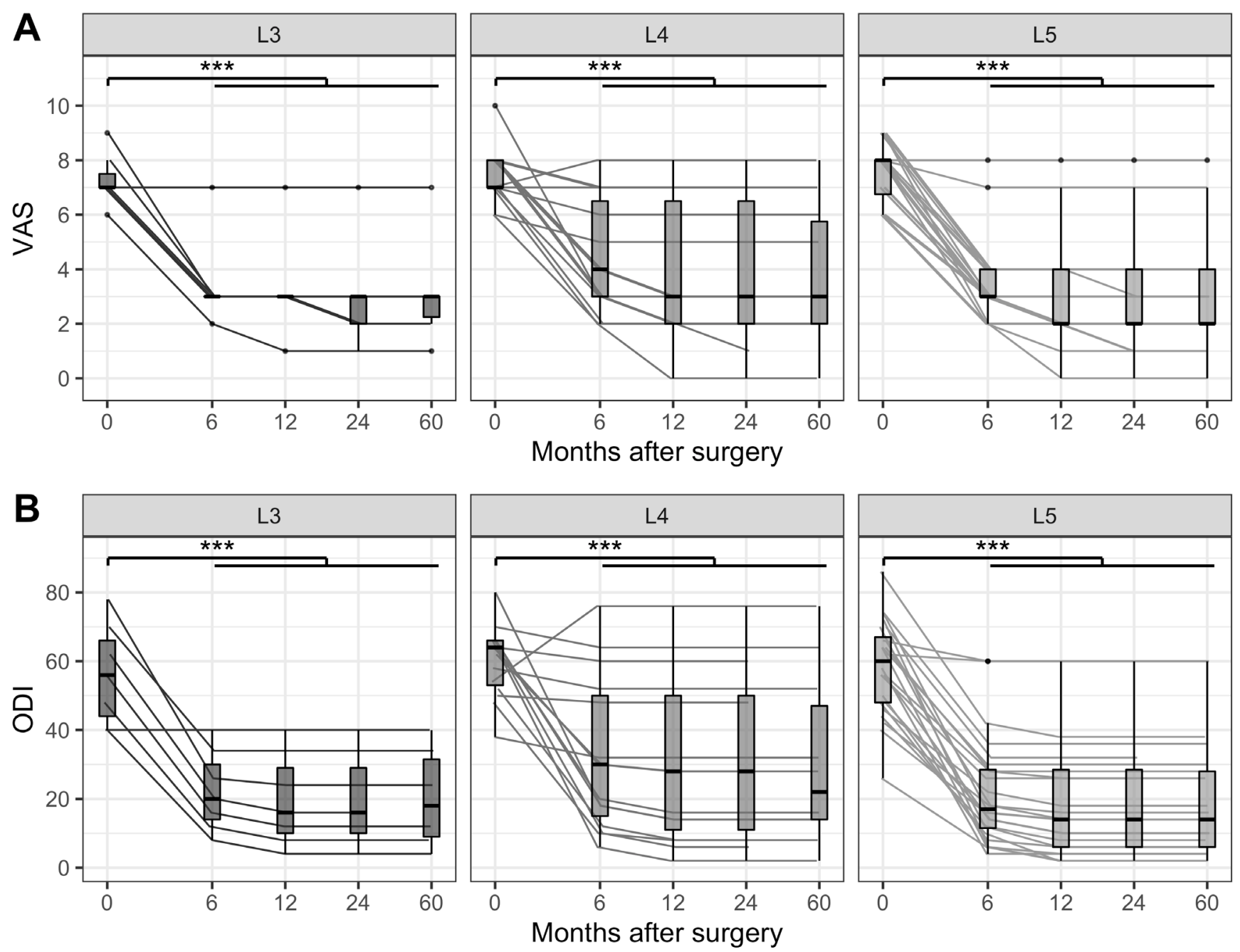

Figure 7. The visual analog scale (VAS) for pain (A) and the Oswestry Disability Index (ODI) (B) measurements in sampled timepoints. Measurements on the same subject were connected with lines. Measurements were also summarized with boxplots at each timepoint. Lower and upper box limits represent the first quartile $\left(Q^{1}\right)$ and third quartile $\left(Q^{3}\right)$ while the thick line inside the box represents the median. Whiskers are connecting minimum or maximum values within the inner fence calculated as $1.5 \times$ interquartile range (calculated as $Q^{3}-Q^{1}$ ) below $Q^{1}$ or above $Q^{3}$, respectively. Any measurements that are not within the inner fence are considered outliers and are represented with points. Statistically significant differences (resulting from post hoc tests) are presented above compared groups with asterisks according to following $P$ value pattern: $0.05>^{*}>0.01>^{* \star}>0.001>^{* \star *}$.

A total of 24 patients had stenosis of the L5 foramen, 15 at the L4 foramen, and 7 at the L3 foramen. Six of the 9 patients who failed to achieve at least a $50 \%$ decrease in outcome scores had stenosis at the L4/5 level, which was somewhat surprising, given the fact that approach to the foramen L5 is always more technically demanding with the iliac crest and transverse process being the major obstacles. Patients with "poor" outcome scores did not have statistically significant differences in preoperative VAS and ODI scores compared to those with favorable outcome. There was also no statistically significant difference in age compared to patients with a good outcome. Six patients among these 9 were men and 3 were women.

The success rates reported for open decompressive surgery, the current gold standard, are between $72 \%$ and
$83 \% .{ }^{10}$ Open surgery, however, requires revision in $8 \%$ of patients at 2 years postoperatively and up to $13 \%$ of cases in the course of 4 years postoperatively. ${ }^{28}$ In the Maine Lumbar Spine Study, 23\% of patients had repeat surgery by 10 years. ${ }^{29}$ In our study, only 1 patient underwent open revision surgery. Revision surgery after an open surgery poses significantly higher risks of further compared to revision surgery after endoscopic foraminoplasty. ${ }^{16}$ After open decompressive surgery, the overall postoperative complication rate was $12 \%$ while $10 \%$ of patients required transfusions intraoperatively and $5 \%$ postoperatively. ${ }^{28}$ The most common surgical complication was dural tear in $9 \%$ of patients. ${ }^{28}$ Postlaminectomy instability and epidural fibrosis rates after open decompressive surgery have been reported to be as high as $25 \%{ }^{30}$ Seymour and $\mathrm{Vaz}^{31}$ 

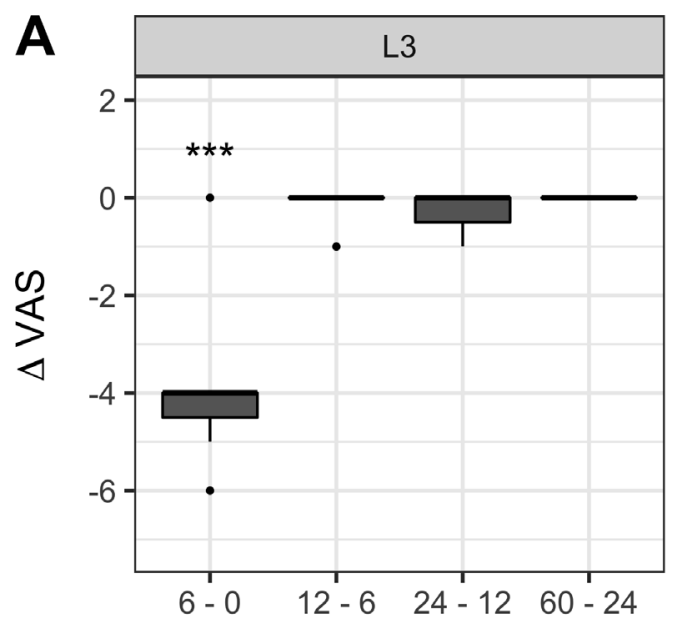
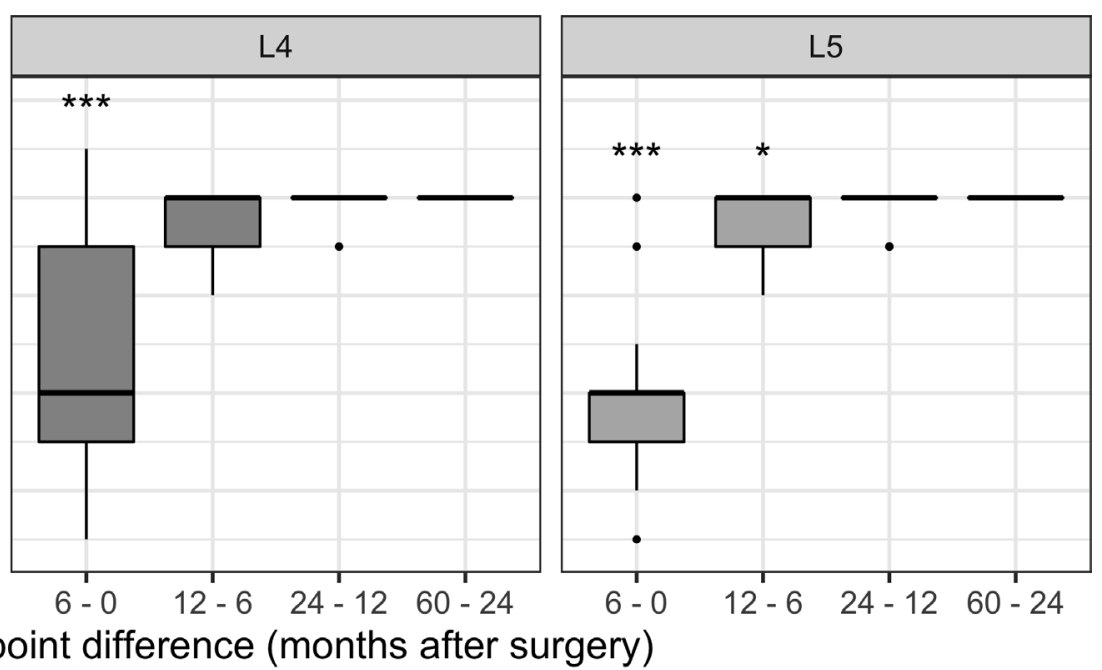

B

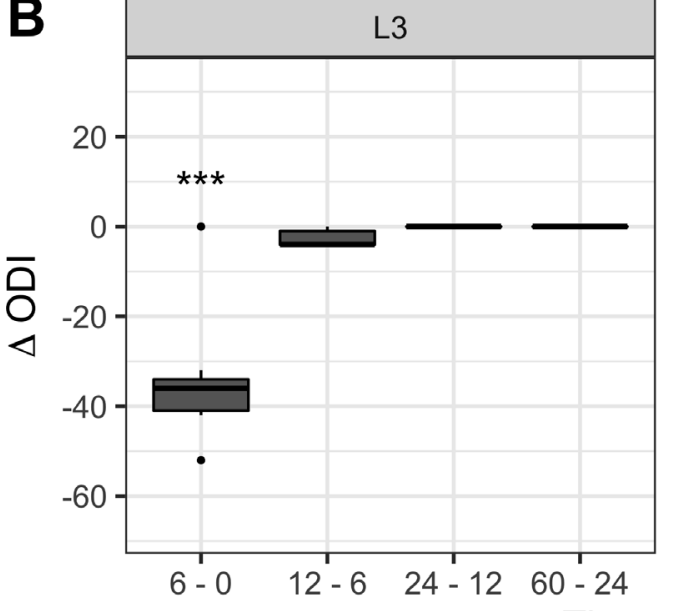

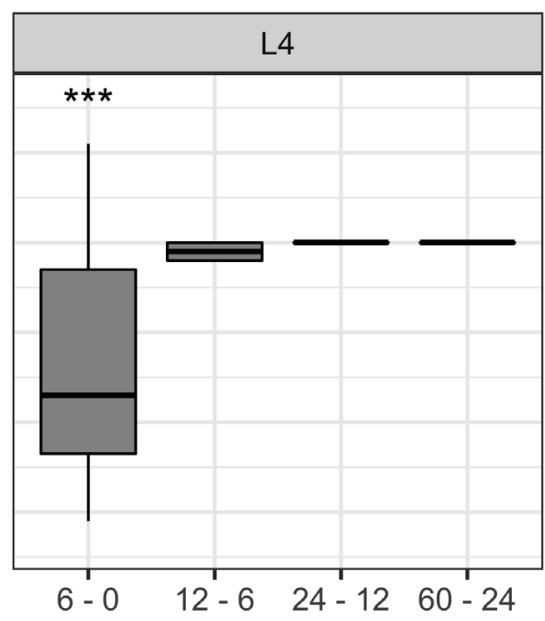

Timepoint difference (months after surgery)

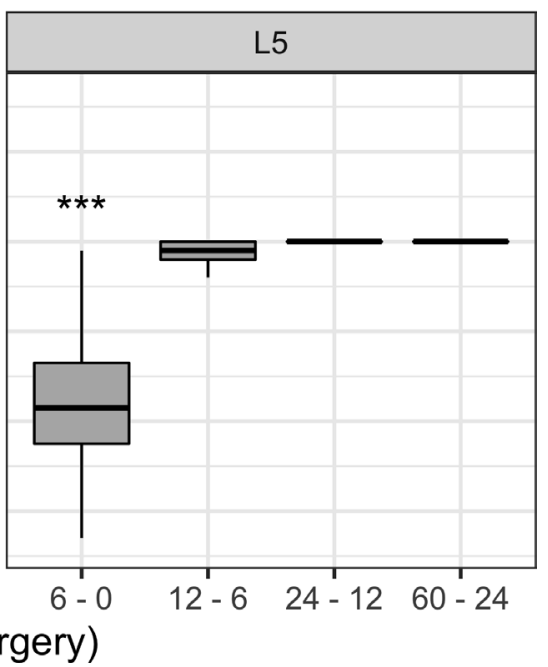

Figure 8. Differences in consecutive timepoints for the visual analog scale (VAS) for pain (A) and the Oswestry Disability Index (ODI) (B). Differences were summarized with boxplots. Lower and upper box limits represent the first quartile $\left(Q^{1}\right)$ and third quartile $\left(Q^{3}\right)$ while the thick line inside the box represents median. Whiskers are connecting minimum or maximum values within inner fence calculated as $1.5 \times$ interquartile range (calculated as $Q^{3}-Q^{1}$ ) below $Q^{1}$ or above $Q^{3}$, respectively. Any measurements that are not within inner fence are considered outliers and represented with points. Statistically significant differences (resulting from post hoc tests) are presented above boxplots with asterisks according to the following $P$ value pattern: $0.05>{ }^{*}>0.01>{ }^{* \star}>0.001>{ }^{* \star *}$.

analyzed 288 cases who had surgeries under general anesthesia aged over 65 . They found postoperative complications in about $60 \%$ of cases. Respiratory complications were the commonest with $17 \%$ of patients having simple atelectasis, $12 \%$ acute bronchitis, and $10 \%$ pneumonia. In this article, postoperative delirium occurred in $7 \%$ of patients, whereas Neufeld et al reported more than $30 \%$ of the postoperative delirium following the general anesthesia in patients aged older than 70 years. ${ }^{31,32}$ The hospital stay is significantly higher in patients who underwent open decompressive surgery compared to those who were operated endoscopically. All patients in our study left the hospital within 24 hours. Forty-three of 46 patients returned to their work or a normal lifestyle after endoscopic surgery. Three patients who scored "poor" and could not resume their normal daily activities were retired patients aged 83,71 , and 71 years. The reported rates of postoperative dysesthesia or numbness after open decompression surgery are $6.5 \%$ to $24 \% .{ }^{10}$ In our case series, only 2 patients $(4.3 \%)$ developed transient postoperative dysesthesia that spontaneously resolved within 4 weeks. These findings highlight the benefits of endoscopic foraminoplasty performed under local anesthesia and TIVA.

\section{Pros and Cons for Transforaminal Endoscopic Foraminoplasty}

A percutaneous approach to the stenotic neuroforamen may be a challenging task for spine surgeons because of the high risk of neural damage to the exiting nerve root. ${ }^{11}$ The safe zone is bordered laterally by the exiting nerve 
root, inferiorly by the end plate or pedicle of the lower vertebra, posteriorly by the SAP of the inferior vertebra, and medially by the traversing nerve root or dura. ${ }^{4}$ It is the authors' opinion that the transforaminal endoscopic approach is better for decompression of single-level foraminal stenosis than the standard open paraspinal approach. There are several reasons for this: a more horizontal access angle to the stenotic foramen, preoperatively planned for each patient, provides a better working field to achieve complete foraminal decompression of the nerve without extensive facetectomy. ${ }^{26}$ The conventional intraspinal approach does not afford clearance of the foraminal pathologies such as the superior foraminal ligament and shoulder or vertebral rim osteophytosis or ensure mobilization of the exiting never root. The skin entry point of the spinal needle is typically located 7-12 $\mathrm{cm}$ lateral from the midline, and its position is extremely important when targeting stenotic foramen of L5. If the skin incision is too medial, the surgeon will not be able to explore the foramen entirely because of the limited angle of vision, and if the skin incision is too lateral, the needle tip is subject to a blockage by the transverse process or iliac crest or the exiting nerve itself.

Circumscribed removal of the perineural scarring, the superior foraminal ligament, shoulder and vertebral rim osteophytes, the apex of the SAP, and anterior facet joint are needed to decompress the entire exiting nerve root. The facet joint and posterior laminar bone can be preserved, thus the risk of postoperative instability or need for fusion surgery is significantly reduced.

The transforaminal percutaneous approach with $1-\mathrm{cm}$ skin incision and parting rather than cutting muscle fibes minimizes soft tissue damage and so reduces the formation of postoperative scar tissue. This is important if additional surgery has to be performed using the same trajectory. A short operative time with reduced blood loss may enhance postoperative recovery and ensures a faster return to work and normal everyday activities. The dorsal root ganglion is mobilized with minimal disturbance, thus reducing the risk of postoperative dysesthesia compared to that in open surgery. ${ }^{10}$ Finally, effective foraminal decompression can be achieved without general anesthesia using only local anesthesia and conscious sedation.

The term foraminoplasty was coined by a coauthor ${ }^{16,19,20,33}$ to differentiate the technique from foraminotomy, which seeks merely to enlarge the bony foramen. By contrast, foraminoplasty addresses not only the optimization of the bony foraminal volume but focuses on restoring the mobility of the exiting nerve root and correction of adverse pathology in and around the foramen. This consists of removal of perineural scarring from pedicle to pedicle and the superior foraminal ligament, facet joint overgrowth, and osteophytes of the facet joint, vertebral rim, and vertebral "shoulder" osteophytes, granulations, and perineural scarring within the safe working zone. The technique ensures that the exiting nerve is mobilized from the vertebra and disc wall and the transiting nerve is mobilized from the medial surface of the facet joint. Foraminoplasty focuses on liberating and mobilizing exiting and descending nerve roots from the epidural space to beyond the external boundaries of the foramen. Thereby, it removes the factors that irritated, distorted, or compressed the nerve roots and caused the pain, reproduced during foraminal palpation, responsible for the patient's predominant presenting symptoms. ${ }^{16}$

According to Sairyo et al, ${ }^{34}$ all the SAP must be removed during foraminotomy to adequately decompress the exiting nerve root. In our opinion, sufficient decompression of a single-level foraminal stenosis can be achieved with more discrete resection of the tip and the cranial anterolateral portion of the SAP. This approach minimizes chances of causing spinal instability while still allowing complete nerve decompression. In cases of combined foraminal and lateral recess stenosis, we believe that the medial part of the foramen must be undercut to improve both foraminal and lateral recess compromise. Kashima et $\mathrm{al}^{35}$ have showed that the SAP facet contact area for the lower lumbar levels is around 50\% and that this decreases with loss of disc height. Therefore, the restricted resection of the anterior facet joint rim optimizes the preservation of the natural contact area in patients with severe foraminal stenosis.

The limitation to this method is the fact that most spine surgeons are still unfamiliar with endoscopic techniques. The learning curve of this surgical technique takes a significant time to achieve maximum efficacy due to the confined access arising from the bone anatomy, especially at foramen L5.

\section{Complications}

Lewandrowski reported an incidence of incidental durotomies in $0.1 \%$ of cases, immediate postoperative foot drop in $0.1 \%$ of cases, spinal headache in $0.4 \%$ of cases, and extravasations of irrigation fluid into the subcutaneous tissues in $3.8 \%$ of cases. ${ }^{25}$ In the article by Ahn et al, transient postoperative dysesthesia was reported in $6.1 \% .^{10}$ In our study, our only complication was transient postoperative dysesthesia in 2 patients, and it spontaneously resolved within 4 weeks of surgery. These short-lived symptoms are most likely due to irritation of the nerve in the narrow confines of the stenotic spinal foramen. A single case of open 
surgery after endoscopic discectomy was also reported by Ahn et $\mathrm{al}^{10}$ in their series of 33 patients. During surgery, no significant complications occurred. There were no cases of disc or wound infection, deep venous thrombosis, dural tears, postoperative foot drop, or spinal headache.

\section{Accessibility}

It is a generally held opinion among nonendoscopic surgeons that the L5 foramen is hardly accessible with the endoscope using transforaminal route due to the transverse process or the iliac crest. Our results, however, clearly show that there is no statistically significant difference in postoperative outcome after sufficient decompression and foraminoplasty regarding the level of foraminal stenosis. The worst results, however, were noted at the L4/L5 level while excellent results were achieved at L5/S1, which exemplifies the fact that large amounts of medial bone do not need to be removed, just mobilization of the structures.

\section{Study Strengths and Weaknesses}

Our study has several limitations. This study was retrospective with a relatively small number of patients. There was no control group in our study, thus preventing the ability to elucidate the effectiveness of endoscopic foraminoplasty compared to conventional open surgery. Strengths included a long follow-up period of 24 months completed by all 46 patients and 60 months follow-up period completed by 37 patients using 2 different outcome measurement tools. Also, the continuity of patient review by an experienced neurosurgeon not directly involved in endoscopic surgery reduced the assessment bias.

\section{CONCLUSION}

Percutaneous transforaminal endoscopic foraminoplasty in patients with single-level foraminal stenosis with long-lasting leg pain resistant to conservative treatment was effective in reducing pain and disability as measured by the VAS and ODI scores. Surgery under local anesthesia, conscious sedation, and circulating analgesia provided added safety for all and especially older patients with significant comorbidities. Once established, the benefits were consistently sustained at 5 years following surgery without evidence of decline in 37 of 46 patients, and the procedure was free of any major complications.

\section{REFERENCES}

1. Nellensteijn J, Ostelo R, Bartels R, Peul W, van Royen B, van Tulder M, et al. Transforaminal endoscopic surgery for lumbar stenosis: a systematic review. Eur Spine J. 2010;19(6):879-886. doi:10.1007/s00586-009-1272-6

2. Ruetten S, Komp M, Merk H, Godolias G. Surgical treatment for lumbar lateral recess stenosis with the full-endoscopic interlaminar approach versus conventional microsurgical technique: a prospective, randomized, controlled study. J Neurosurg Spine. 2009;10(5):476-485. doi:10.3171/2008.7.17634

3. Ruetten S, Komp M, Hahn P, Oezdemir S. Decompression of lumbar lateral spinal stenosis: full-endoscopic, interlaminar technique. Oper Orthop Traumatol. 2013;25(1):31-46. doi:10.1007/ s00064-012-0195-2

4. Kambin P, Casey K, O’Brien E, Zhou L. Transforaminal arthroscopic decompression of lateral recess stenosis. J Neurosurg. 1996;84(3):462-467. doi:10.3171/jns.1996.84.3.0462

5. Kobayashi S. Pathophysiology, diagnosis and treatment of intermittent claudication in patients with lumbar canal stenosis. World J Orthop. 2014;5(2):134-145. doi:10.5312/wjo.v5.i2.134

6. Zochodne DW. Epineurial peptides: a role in neuropathic pain? Can J Neurol Sci. 1993;20(1):69-72. doi:10.1017/ s0317167100047466

7. Teixeira MJ, Almeida DB, Yeng LT. Concept of acute neuropathic pain. The role of nervi nervorum in the distinction between acute nociceptive and neuropathic pain. Revista Dor. 2016;17(Suppl 1):S5-10. doi:10.5935/1806-0013.20160038

8. Chang SB, Lee SH, Ahn Y, Kim JM. Risk factor for unsatisfactory outcome after lumbar foraminal and far lateral microdecompression. Spine. 2006;31(10):1163-1167. doi:10.1097/01.brs. 0000216431.69359 .91

9. Ahn Y, Lee SH, Park WM, Lee HY. Posterolateral percutaneous endoscopic lumbar foraminotomy for L5-S1 foraminal or lateral exit zone stenosis. Technical note. J Neurosurg. 2003;99(3 Suppl):320-323. doi:10.3171/spi.2003.99.3.0320

10. Ahn Y, Oh H-K, Kim H, Lee S-H, Lee H-N. Percutaneous endoscopic lumbar foraminotomy: an advanced surgical technique and clinical outcomes. Neurosurgery. 2014;75(2):124-133. doi:10.1227/NEU.0000000000000361

11. Wildermuth S, Zanetti M, Duewell S, et al. Lumbar spine: quantitative and qualitative assessment of positional (upright flexion and extension) MR imaging and myelography. Radiology. 1998;207(2):391-398. doi:10.1148/radiology.207.2.9577486

12. Lee S, Lee JW, Yeom JS, et al. A practical MRI grading system for lumbar foraminal stenosis. AJR Am J Roentgenol. 2010;194(4):1095-1098. doi:10.2214/AJR.09.2772

13. Park H-J, Kim SS, Lee S-Y, et al. Clinical correlation of a new MR imaging method for assessing lumbar foraminal stenosis. AJNR Am J Neuroradiol. 2012;33(5):818-822. doi:10.3174/ajnr. A2870

14. Knight MTN, Krishnan A. Spinal surgery. In: Whitwam JG, McLoy RF, eds. Principles and Practice of Sedation. Oxford: Blackwell Science; 1998:178-181.

15. Kambin P, Sampson S. Posterolateral percutaneous suctionexcision of herniated lumbar intervertebral discs. Clin Orthop Relat Res. 1986;207(amp;NA):37. doi:10.1097/00003086-19860600000008

16. Knight MTN, Jago I, Norris C, Midwinter L, Boynes C. Transforaminal endoscopic lumbar decompression \& foraminoplasty: a 10 year prospective survivability outcome study of the 
treatment of foraminal stenosis and failed back surgery. Int J Spine Surg. 2014;8:21. doi:10.14444/1021

17. Ahn Y. Percutaneous endoscopic decompression for lumbar spinal stenosis. Expert Rev Med Devices. 2014;11(6):605-616. doi: 10.1586/17434440.2014.940314

18. Okuda T, Fujimoto Y, Tanaka N, Ishida O, Baba I, Ochi M. Morphological changes of the ligamentum flavum as a cause of nerve root compression. Eur Spine J. 2005;14(3):277-286. doi:10.1007/s00586-004-0782-5

19. Knight MT, Goswami A, Patko JT, Buxton N. Endoscopic foraminoplasty: a prospective study on 250 consecutive patients with independent evaluation. J Clin Laser Med Surg. 2001;19(2):73-81. doi:10.1089/104454701750285395

20. Knight MT, Vajda A, Jakab GV, Awan S. Endoscopic laser foraminoplasty on the lumbar spine--early experience. Minim Invasive Neurosurg. 1998;41(1):5-9. doi:10.1055/s-2008-1052006

21. Ng LCL, Tafazal S, Sell P, Ng LC. The effect of duration of symptoms on standard outcome measures in the surgical treatment of spinal stenosis. Eur Spine J. 2007;16(2):199-206. doi:10.1007/ s00586-006-0078-z

22. Ostelo RWJG, Deyo RA, Stratford P, et al. Interpreting change scores for pain and functional status in low back pain: towards international consensus regarding minimal important change. Spine. 2008;33(1):90-94. doi:10.1097/BRS. 0b013e31815e3a10

23. Yeung A, Gore S. Endoscopic foraminal decompression for failed back surgery syndrome under local anesthesia. Int J Spine Surg. 2014;8. doi:10.14444/1022

24. Lewandrowski KU. "Outside-in" technique, clinical results, and indications with transforaminal lumbar endoscopic surgery: a retrospective study on 220 patients on applied radiographic classification of foraminal spinal stenosis. Int J Spine Surg. 2014;8. doi: $10.14444 / 1026$

25. Lewandrowski KU. Readmissions after outpatient transforaminal decompression for lumbar foraminal and lateral recess stenosis. Int J Spine Surg. 2018;12(3):342-351. doi:10.14444/5040

26. Ahn Y, Keum HJ, Son S. Percutaneous endoscopic lumbar foraminotomy for foraminal stenosis with postlaminectomy syndrome in geriatric patients. World Neurosurg. 2019;130:e1070-e1076. doi:10.1016/j.wneu.2019.07.087

27. Knight Met al. A radiofrequency treatment pathway for cluneal nerve disorders. EC Orthop. 2020;11(3):1-19.

28. Weinstein JN, Tosteson TD, Lurie JD, et al. Surgical versus nonsurgical therapy for lumbar spinal stenosis. $N$ Engl J Med. 2008;358(8):794-810. doi:10.1056/NEJMoa0707136

29. Atlas SJ, Keller RB, Wu YA, Deyo RA, Singer DE. Longterm outcomes of surgical and nonsurgical management of lumbar spinal stenosis: 8 to 10 year results from the maine lumbar spine study. Spine. 2005;30(8):936-943. doi:10.1097/01.brs.0000158953. 57966.c0

30. Mullin BB, Rea GL, Irsik R, Catton M, Miner ME. The effect of postlaminectomy spinal instability on the outcome of lumbar spinal stenosis patients. J Spinal Disord. 1996;9(2):107-116. doi:10.1097/00002517-199604000-00004

31. Seymour DG, Vaz FG. A prospective study of elderly general surgical patients: II. Post-operative complications. Age Ageing. 1989;18(5):316-326. doi:10.1093/ageing/18.5.316

32. Neufeld KJ, Leoutsakos JS, Sieber FE, et al. Evaluation of two delirium screening tools for detecting post-operative delirium in the elderly. Br J Anaesth. 2013;111(4):612-618. doi:10.1093/bja/ aet167
33. Knight M, Goswami A. Management of isthmic spondylolisthesis with posterolateral endoscopic foraminal decompression. Spine. 2003;28(6):573-581. doi:10.1097/01. BRS.0000050400.16499.ED

34. Sairyo K, Chikawa T, Nagamachi A. State-of-the-art transforaminal percutaneous endoscopic lumbar surgery under local anesthesia: discectomy, foraminoplasty, and ventral facetectomy. $J$ Orthop Sci. 2018;23(2):229-236. doi:10.1016/j.jos.2017.10.015

35. Kashima M, Chikawa T, Matsumura M, et al. Morphological analysis of the intervertebral foramen for the safer performance of TF-PELD and TF-PEVF. In: The 17th Annual Meeting of the Pacific and Asian Society of the Minimally Invasive Spine Surgery. Sapporo Japan; 2017.

Funding: The author(s) received no financial support for the research, authorship, and/or publication of this article.

Declaration of Conflicting Interests: All authors certify that they have no affiliations with or involvement in any organization or entity with any financial interest (such as honoraria; educational grants; participation in speakers' bureaus; membership, employment, consultancies, stock ownership, or other equity interest; and expert testimony or patent-licensing arrangements) or nonfinancial interest (such as personal or professional relationships, affiliations, knowledge, or beliefs) in the subject matter or materials discussed in this manuscript.

Patient Consent for Publication: Informed consent was obtained from all individual participants included in the study.

Ethics Approval: All procedures performed in our study involving human participants were in accordance with the ethical standards of the institutional research committee (Aksis Specialty hospital for Spine and Orthopaedic surgery) and with the 1964 Helsinki declaration and its later amendments or comparable ethical standards.

Data Availability Statement: All data analyzed during this study are included in this published article.

Corresponding Author: Karlo Houra, Aksis Specialty Hospital for Spine and Orthopaedic Surgery, Petrovaradinska 1, 10000 Zagreb, Croatia; khoura@ unin.hr

Published 15 February 2022

This manuscript is generously published free of charge by ISASS, the International Society for the Advancement of Spine Surgery. Copyright (c) 2022 ISASS. To see more or order reprints or permissions, see http:// ijssurgery.com. 